\title{
A highly collimated jet from the low mass proto-star NGC1333 IRAS 4B
}

\author{
J.-F. Desmurs* \\ Observatorio Astronómico Nacional, Spain \\ E-mail: desmurseoan.es \\ C. Codella \\ IRA, INAF, Italy \\ E-mail: codelladarcetri.astro.it

\section{J. Santiago-García} \\ Observatorio Astronómico Nacional, Spain \\ E-mail: j.santiagodoan.es

\section{Tafalla} \\ Observatorio Astronómico Nacional, Spain \\ E-mail: tafalladoan.es

\section{R. Bachiller} \\ Observatorio Astronómico Nacional, Spain \\ E-mail: r.bachiller@oan.es
}

We imaged the protostars of the nearby region NGC1333 IRAS 4 in the water maser line at 22.2 $\mathrm{GHz}$ by using the VLBA in phase referencing at milliarcsecond scale over four epochs spaced by one month to measure proper motions. We measure the absolute positions and proper motions of the $\mathrm{H}_{2} \mathrm{O}$ spots to investigate the kinematics of the region from where the jet is launched.

Two protostars (A2 and B) have been detected in a highly variable $\mathrm{H}_{2} \mathrm{O}$ maser emission, with an active phase shorter than four weeks. A $70 \mathrm{AU}$ chain of several maser spots, very well aligned, has been observed close to the B protostar. The apparent proper motions have been derived, finding that the $\mathrm{H}_{2} \mathrm{O}$ spots are moving along the N-NW direction with projected velocities between 10 and $50 \mathrm{~km} \mathrm{~s}^{-1}$. We conclude that in IRAS 4B, water masers trace a highly collimated bipolar jet clearly associated with the protostar.

The 9th European VLBI Network Symposium on The role of VLBI in the Golden Age for Radio Astronomy and EVN Users Meeting

September 23-26, 2008

Bologna, Italy

\footnotetext{
* Speaker.
} 


\section{Introduction}

NGC1333 is a well-known star forming region containing a large number of proto-stars and located at a distance of $235 \mathrm{pc}$, according to the recent observations [2] performed with VERA (VLBI Exploration of Radio Astrometry). The IRAS 4 region contains three star forming sites, called $4 \mathrm{~A}, 4 \mathrm{~B}$, and $4 \mathrm{C}$, which can be identified in the mm-continuum. High angular resolution $\mathrm{cm}$ - and mm-observations [6] have revealed that IRAS 4B is also associated with a bipolar outflow located along the North-South direction [3]. Water $\left(\mathrm{H}_{2} \mathrm{O}\right)$ masers at $22 \mathrm{GHz}$ in combination with the high angular resolution given by Very Long Baseline Interferometry (VLBI) techniques, represent a unique tool to investigate the mechanism of jet formation and collimation in the earliest star forming phases. All these characteristics make of IRAS4 an excellent laboratory where to study multiple star formation.

\section{Observations and Results}

Using the VLBA network, we observed $\mathrm{H}_{2} \mathrm{O}$ maser emission at $22.2 \mathrm{GHz}$ in phase referencing over four epochs spaced by about four weeks during 2003 (Apr 1st, May 14th, June 11th, July 07th). The duration of each observations was 10 hours in total.

As the four proto-stars (IRAS 4A1, 4A2, 4B, and 4C) fell inside the primary beam of the antennas, during the observation we tracked their barycenter. Data were recorded in dual circular polarization with a velocity resolution (i.e. channel width) of $\sim 0.1 \mathrm{~km} \mathrm{~s}^{-1}$ and a total velocity coverage of about $55 \mathrm{~km} \mathrm{~s}^{-1}$. To be able to measure and compare the relative position of the water maser emission across the epochs, we used the phase referencing technique with the close phase calibrator $0333+321$ (1.5 degree away). Data were correlated in three separate passes, one per source, at the VLBA correlator in Socorro (New Mexico, USA) with an integration time of 2 seconds. The data reduction was performed using the Astronomical Image Processing System (AIPS) package. The amplitude was calibrated using the template spectra method. The final restoring beam was $1.2 \times 1.2$ mas and each channel was cleaned down to a residual rms noise of $\sim 15 \mathrm{mJy}$ per beam.

In the first epoch, no emission has been detected towards the four sources. The proto-stars IRAS 4A1+4A2 and IRAS 4B have shown water emission in two of the four epochs observed. This confirms the high variability of the $\mathrm{H}_{2} \mathrm{O}$ spots associated with low-mass young stellar objects, with an active phase shorter than a few weeks.

Figure 1 reports the July 07th map for IRAS 4B with two groups of $\mathrm{H}_{2} \mathrm{O}$ spots mapped: redshited emission at NW $\sim[+12 /+17.0] \mathrm{km} \mathrm{s}^{-1}$, blueshited at $\mathrm{SE} \sim 0 \mathrm{~km} \mathrm{~s}^{-1}$ (ambient emission is at $\left.V_{\mathrm{LSR}}=+7.25 \mathrm{~km} \mathrm{~s}^{-1}\right)$. The size of the water spots chain is about 270 mas $(\sim 60 \mathrm{AU}$ at $235 \mathrm{pc})$ and its position angle is P.A. $\sim 25^{\circ}$ NW. As a reference, the dashed circle in Fig. 1 reports the coordinates of IRAS B following [6] who gave the position of the NGC1333 proto-stars, observed at 3.6 $\mathrm{cm}$, with a 50 mas uncertainty (size of the circle). The closest spot to IRAS B, being detected at the level of the uncertainty area, is at $\sim 10 \mathrm{AU}$. The very good alignment of the maser spots as well as their velocities suggest their association with a molecular jet driven by the IRAS B proto-star and having a high degree of collimation (this is consistent with EVN observations of this source by [1]). If we conservatively assume an inclination angle $\simeq 20^{\circ}-30^{\circ}$, we derive that the water masers 


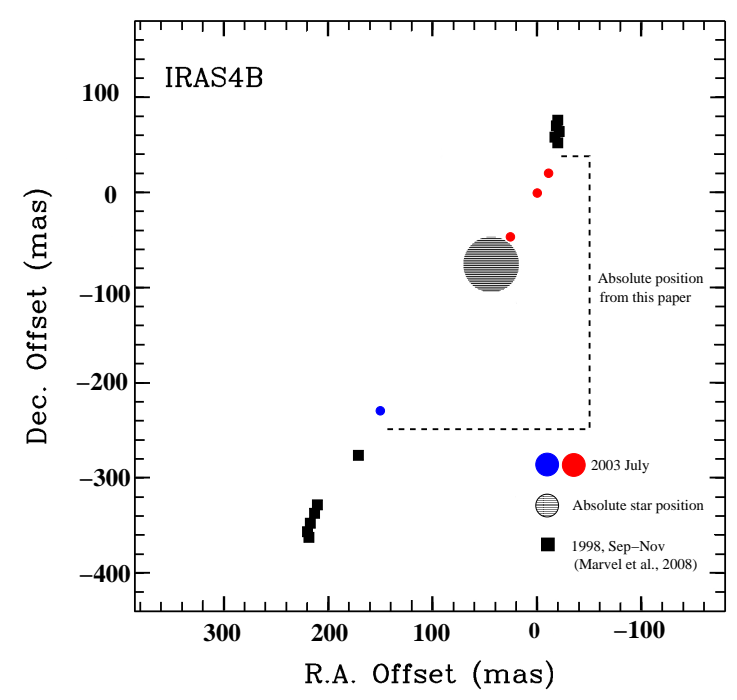

Figure 1: Figure 1. Water maser maps measured for IRAS 4B (July 07th). The map reports the chain of $\mathrm{H}_{2} \mathrm{O}$ spots distributed over $\sim 70 \mathrm{AU}$. The driving protostar (hash circle; [6]) is at $+66,-95$ mas with respect to the center of the present maps, at $\alpha_{2000}=03^{\mathrm{h}} 29^{\mathrm{m}} 12^{\mathrm{s}} 003, \delta_{2000}$ $=+31^{\circ} 13^{\prime} 08^{\prime \prime} 14$. The size of the circle stands for the uncertainty (50 mas) associated with the position of the proto-star. Black squares report the water masers observed by [5], their locations are just indicative with respect to our results.

are moving towards the N-NW (Red) and the S-SE (Blue) direction with velocities $V_{\mathrm{H}_{2} \mathrm{O}} \simeq 20-150$ $\mathrm{km} \mathrm{s}^{-1}$, in agreement with the high velocities usually observed along jets form proto-stars.

Our observations are consistent with those recently published by [5] using VLBA observations from 1998 from water masers associated with NGC 1333-IRAS4B. The orientation of the jet traced by the water maser emission has nearly the same position angle (observations of the two projects were separated by five years), and shows high velocities but does not seem to coincide with the large scale outflow located along the $\mathrm{N}-\mathrm{S}$ direction.

Further observations at high angular resolution of the molecular outflow are needed to clarify whether the disagreement between the molecular outflow and the water jet is due to precession or whether we simply observe different components driven by different sources.

\section{References}

[1] J.-F. Desmurs, C. Codella, M. Tafalla \& R. Bachiller, 2000, Preliminary results on water maser EVN observations and the collimation of protostellar outflows EVN Symposium 2000, Proceedings of the 5th European VLBI Network Eds.: J.E. Conway, A.G. Polatidis, R.S. Booth and Y.M. Pihlström, published Onsala Space Observatory, 183.

[2] T. Hirota, T. Bushimata, Y.K. Choi, et al., 2008,Astrometry of H2O Masers in Nearby Star-Forming Regions with VERA II SVS13 in NGC1333 PASJ 60, 37 [astro-ph/0709.1626v2].

[3] J.K. Jørgensen, T.L. Bourke, P.C. Myers, et al., 2007, PROSAC: A Submillimeter Array Survey of Low-Mass Protostars. I. Overview of Program: Envelopes, Disks, Outflows, and Hot Cores ApJ 659, 479-498 [astro-ph/0701115]

[4] L.S.G. Meehan, B.A.Wilking, M.J. Claussen, L.G. Mundy, \& A. Wootten, 1998, Water Masers in the Circumstellar Environments of Young Stellar ObjectsAJ 115, 1599.

[5] K.B. Marvel, B.A. Wilking, M.J. Claussen, \& A. Wootten, 2008, Time-Resolved AU-Scale Jets Traced by Masers in the IRAS 4A/B Regions of NGC 1333 ApJ 685, 285 [astro-ph/0808.0171]

[6] B. Reipurth, L.F. Rodríguez, G. Anglada \& J. Bally, 2002, Radio Continuum Maps of Deeply Embedded Protostars: Thermal Jets, Multiplicity, and Variability AJ 124, 1045. 